\title{
The Effects of the Supplementation with Snakehead Fish and Sea Cucumber Extracts on Platelet-Derived Growth Factor in Post-Operative Patients
}

\author{
Otniel Wendy Wahono, JB. Suparyatmo, Dian Ariningrum \\ Department of Clinical Pathology, Faculty of Medicine, Sebelas Maret University/Dr. Moewardi Hospital, Surakarta, Indonesia. \\ E-mail:otnielwahono@gmail.com
}

\begin{abstract}
Wound healing is a complex process that involves the interaction between Extracellular Matrix (ECM) cells and mediated by cytokines and various growth factors, especially platelet-derived growth factor (PDGF) which plays a role in almost every phase of wound healing. Applying snakehead fish and sea cucumber extract containing amino acids and fatty acids in post-operative patients is expected to increase PDGF levels and enable the acceleration of the wound healing process. This study was a double-blind randomized control trial with a pre- and post-treatment approach, which involved 34 post-operative patients divided into two groups; the control group (K1) and treatment group (K2) and was carried out from April to June 2019 at Dr. Moewardi Hospital in Surakarta. Independent sample T-test was used for data with normal distribution to determine the mean differences between the control and treatment groups, while the Mann-Whitney test was used for data with the abnormal distribution. Also, to determine the mean difference before and after the treatment of one paired group, a paired T-test was used for data with normal distribution, while the Wilcoxon test was used for data with the abnormal distribution. $p<0.05$ was significant with $95 \%$ Confidence Intervals (CI). The supplementation of snakehead fish and sea cucumber extract significantly increased PDGF levels in the treatment group (K2) compared to the controls group (K1) ( $p<0.001)$. This study findings showed that snakehead fish and sea cucumber extract were able to increase PDGF levels in post-operative patients on the fourth day.
\end{abstract}

Keywords: Snakehead fish and sea cucumber extract, PDGF, surgical wound healing

\section{INTRODUCTION}

Wound healing is a complex process that involves interactions between cells and the Extracellular Matrix (ECM). Wound healing is mediated by cytokines and various growth factors. Simultaneous and sequential wound healing involves hemostasis, inflammation, cell proliferation, ECM production, fibroplasia, and wound contraction. Many factors are known to influence wound healing. The role of growth factors has been widely studied because it plays an important role in regulating cell behavior, elaboration, and ECM remodeling. In-vitro, various growth factors such as Epidermal Growth Factor (EGF), Vascular Endothelial Growth Factor (VEGF), Insulin-like Growth Factor (IGF), Nerve Growth Factor (NGF), Platelet-Derived Growth Factor (PDGF) each plays a role in stimulating the cell proliferation, migration, synthesis, and degradation of the extracellular matrix. Furthermore, when applied exogenously in-vivo, these factors can also promote wound healing. ${ }^{1}$

Some studies identify cellular processes and the contributing components associated with wound healing, such as platelets, neutrophils, macrophages, and fibroblasts. Those components release several cytokines including interleukin (IL-1, IL-6) and tumor necrosis factor- $\alpha$ (TNF- $\alpha$ ), and growth factors such as PDGF which are thought to play an important role. ${ }^{2}$ In the hematopoietic system, PDGF is synthesized in megakaryocyte granules and stored in platelet a-granules secreted during platelet aggregation. Other cells that produce PDGF are macrophages, endothelial cells, fibroblasts, vascular smooth muscle cells, osteoblasts, glia, and neurons. ${ }^{3}$

Wound healing is classically divided into four overlapping stages: hemostasis, inflammation, proliferation, and remodeling. Each stage is characterized by key molecular, cellular, and physiologic events which are orchestrated in large part by signaling among hematopoietic, immunologic, and resident skin cells. Immediately after a skin injury, multiple physiologic responses are triggered to stop blood loss. Local vascular smooth muscle cells constrict vessels to reduce blood flow. Platelets and coagulation cascade factors form a hemostatic fibrin clot, which serves as a scaffold for the migration of cells, including leukocytes, 
keratinocytes, and fibroblasts, into the wound. The inflammatory stage is initiated within hours after injury and is fueled by platelet-derived mediators, bacterial by-products, and secreted chemoattractants. Neutrophils formerly infiltrate the injury site, kill bacteria and degrade damaged matrix proteins. Monocytes arrive within 24 hours and transform into macrophages to kill microbes, remove tissue debris, destroy remaining neutrophils, and pave the way for angiogenesis and tissue granulation. Macrophages also assist in the transition to the proliferative stage, a process whereby newly produced cells fill the wound defect, by releasing a set of growth factors and chemokines including Platelet-Derived Growth Factor (PDGF), Fibroblast Growth Factors (FGFs), Vascular Endothelial Growth Factor (VEGF), and Transforming Growth Factor-a and -b (TGFa and TGFb), which induce cell migration, cell proliferation, and matrix formation. Stem cell reservoirs in the hair follicle bulge, isthmus, and interfollicular epidermis release keratinocytes, which proliferate and migrate to achieve wound coverage, then undergo stratification and differentiation to rebuild the epidermal barrier. In concert with epidermal repair, angiogenesis begins, stimulated by multiple growth factors including VEGF and FGF-2. The admixture of newly formed blood vessels with fibroblasts, macrophages, and matrix proteins forms "granulation tissue," the soft and pink material that appears at the base of a healing wound. Toward the end of the proliferative stage, fibroblasts differentiate into actin-rich, contractile myofibroblasts, which pull together the wound edges. The remodeling phase involves a transition of the dermis from type III to type I collagen predominance, in concert with the removal of cells from earlier stages. Collagen remodeling involves Matrix Metalloproteinases (MMPs) and altered collagen synthesis to produce a scar. The tensile strength of wounded skin increases during this phase, regaining $40 \%$ of its original strength at $1^{\text {st }}$ month and $70 \%$ by one year. Failure to initiate, terminate, or regulate any particular stage results in pathologic wound healing and manifests in cutaneous entities such as pyogenic granulomas (overgrowth of granulation tissue), hypertrophic scars and keloids (excessive fibrotic response), or chronic ulcers (prolonged inflammation and inability to re-epithelialize). ${ }^{4}$

Many PDGF concentrations in platelets and fluid are produced during the initial stages of wound healing. ${ }^{5}$ Normal levels of PDGF in human serum are $273 \pm 25 \mathrm{ng} / \mathrm{mL}$ with a half-life of 24 hours. ${ }^{6.7}$ In this study sandwich ELISA method was used as the gold standard method of PDGF levels measurement. ${ }^{8}$

Channa striata, known as snakehead fish with freshwater habitats found in the Southeast Asian regions, has many benefits because of its protein content and its use in traditional medicine. Snakehead fish extract contains various biochemical components, such as amino acids, especially glycine which is important for the synthesis of collagen fibers during wound healing and fatty acids such as Arachidonic Acid (AA), Eicosapentaenoic Acid (EPA), and Docosahexaenoic Acid (DHA). The AA content in snakehead fish extract can stimulate the synthesis of prostaglandins, which play an important role in wound healing. ${ }^{9}$

Sea cucumber is commonly known as "teripang", has long been used in traditional medicine for Asian and Middle Eastern people. Some unique biological and pharmacological activities such as: anti-angiogenic, anti-cancer, anti-coagulant, anti-hypertensive, anti-inflammatory, anti-microbial, anti-oxidant, anti-thrombotic, anti-cancer, and wound healing have been ascribed to chemical compounds extracted from various species of sea cucumbers. The medicinal benefits of sea cucumber extract are related to the content of essential amino acids and polyunsaturated fatty acids which are key components in tissue repair. The fatty acid profiles include $A A, E P A$, and $D H A$, which are almost similar to the content in fish extract. ${ }^{10}$

The AA content in snakehead fish and sea cucumber fish extract will be metabolized in the human body through two pathways, such as cyclooxygenase and 5-lipooxygenase. In the cyclooxygenase pathway, prostaglandin G2 (PGG2) will be produced through reactions that depend on glutathione hydroperoxide immediately converted into prostaglandin $\mathrm{H} 2(\mathrm{PGH} 2)$, which will produce thromboxane A2 (TXA2), which plays a role in vasoconstriction of blood vessels, the release of platelet- $\alpha$ granules, platelet aggregation and adhesion to endothelial tissue to initiate blood clotting. In the 5-lipooxygenase pathway, leukotriene A4 will be produced and synthesized to produce leukotriene B4, which acts as a chemoattractant for inflammatory cells. ${ }^{11}$

The results of a study by Shafri and Manan proved that the administration of snakehead fish extract was effective in wound healing due to its high amino acid content, such as glycine, a fatty acid involved in several wound healing events from collagen reactions, wound contractions, and tissue re-epithelialization to wounds. ${ }^{12}$ Research by Mohamed et al. proved that sea cucumber extract (Stichopus chloronotus) significantly showed the 
best minor wound healing properties at a concentration of $0.5 \%$ compared to the normal control group and the positive control group. ${ }^{13}$

Administration of snakehead fish and sea cucumber extract containing amino acids and fatty acids in post-operative patients is expected to increase the levels of PDGF which play a role in each phase of wound healing, to shorten the phase of hemostasis and inflammation, continue the proliferation and remodeling phase to speed up the healing process.

\section{METHODS}

The design used in this study was a double-blind randomized control trial with a pre- and post-test approach in two groups consisting of the control group (standard therapy)/K1 and treatment group (standard therapy and the snakehead fish and sea cucumber extracts)/K2. In this study, the initial measurements of PDGF levels were carried out on the first day of post-operative and final measurements were carried out on the fourth day. This research was performed in the Clinical Pathology Laboratory of Dr. Moewardi (RSDM) Hospital in Surakarta on April-June 2019 by involving 34 research subjects who met the inclusion and exclusion criteria and divided into 17 subjects each group.

The inclusion criteria for this study were post-operative patients aged $18-65$ years, willing to take part in the study and signed the informed consent sheet. Exclusion criteria included patients who were allergic to snakehead and sea cucumber extract based on history or medical records and patients with liver function disorders, hematological malignancies, burn wound, diabetes mellitus, chronic renal failure, atherosclerosis, neoplasia, desmoplasia, unstable angina pectoris, thrombocytopenia in dengue fever and history of drugs use that suppress the bone marrow.

Laboratory tests were preceded by conducting analytical precision to account for the results of the examination. The basic characteristics of research subjects were reported as mean and Standard Deviation (SD) if the conditions were met, while data distribution was determined using the Saphiro-Wilks test. To determine the average difference in the K1 group with the K2 group, the independent sample T-test was used for data with the normal distribution, and the Mann-Whitney test was used for data with the abnormal distribution. Also, to determine the difference between the pre and post mean in one group, a paired T-test was used in pairs for data with normal distribution and the Wilcoxon test was used for data with the abnormal distribution. Data were processed using a computer program, and considered significant if $p<0.05$ with a 95\% Confidence Interval (CI).

This study was approved by The Health Research Ethics Committee of the Faculty of Medicine, Universitas Sebelas Maret/Dr. Moewardi Hospital with number 565/IV/HREC/2019.

\section{RESULT AND DISCUSSION}

The results of a day-to-day accuracy test of PDGF levels were good and indicated the persistence of each examination. The basic characteristics of the subjects in this study are shown in Table 1.

Qualitative characteristic variables with categorical scales were gender and surgery, while the quantitative characteristic variables with a numerical scale were age, weight, height, albumin, and BMI levels. To determine the homogeneity of the characteristic variables with categorical and numerical data in the control and the treatment group, the Shapiro-Wilk test was used because the number of research subjects was less than 50 .

The results of the homogeneity test of the gender characteristics showed homogeneity of gender between the control and treatment groups. The $\mathrm{K} 1$ group consisted of 6 male (35.3\%) and 11 female (64.7\%) subjects, while the K2 group consisted of 10 male (58.8\%) and 7 female (41.2\%) subjects. The Chi-Square test showed $p=0.303$ which indicated no difference in the proportion of male and female gender between $\mathrm{K} 1$ and $\mathrm{K} 2$ groups.

Shapiro-Wilks normality test was carried out and showed normal distribution of the data for age, weight, height, and albumin levels. Therefore, the difference test of independent sample T-test was used. Age characteristics showed a mean (SD) of 42.29 (12.11) years in the K1 group. The K2 group showed a mean (SD) of 40.88 (13.02) years. Statistical analysis of the age characteristics showed $p$-value $=0.746$ $(p>0.05)$, indicating no significant difference in age between the two study groups.

Bodyweight characteristics showed a mean (SD) of 56.29 (8.74) kg in the $\mathrm{K} 1$ group and a mean (SD) of 53.00 (6.19) kg in the K2 group. Statistical tests of weight characteristics showed $p=0.215$ ( $p>0.05$ ), indicating no significant difference in body weight between the two study groups. 
Height characteristics showed a mean (SD) of $164.29(3.5) \mathrm{cm}$ in the $\mathrm{K} 1$ group and a mean (SD) of $165.06(3.77) \mathrm{cm}$ in the K2 group. Statistical tests for height characteristics showed $p=0.545$ ( $p>0.05$ ), indicating no significant difference in height between the two study groups.

The characteristics of albumin levels showed a mean (SD) of $2.89(0.53) \mathrm{g} / \mathrm{dL}$ in the $\mathrm{K} 1$ group and a mean (SD) of $2.8(0.51) \mathrm{g} / \mathrm{dL}$ in the K2 group. Statistical tests of albumin levels showed $p=0.622$ $(p>0.05)$, indicating no significant difference in albumin levels between the two study groups.

Homogeneity testing was also performed on other characteristic variables including Body Mass Index (BMI) and surgery. The normality test of Shapiro-Wilks showed an abnormal distribution of BMI data surgery; therefore, the transformation of data using log 10 was carried out. However, after log 10 transformation the data was still not normally distributed, and BMI differences in the two groups were then determined. The Kruskal-Wallis test results showed no significant difference in BMI between the $\mathrm{K} 1$ group and the $\mathrm{K} 2$ group with a $p$-value $=0.543(p>0.05)$, while the Mann-Whitney test showed no significant difference in surgery between K1 group and the K2 group with a $p$-value $=0.897(p>0.05)$.

Platelet-derived growth factor levels before treatment in the $\mathrm{K} 1$ group showed mean (SD) values of 561.55 (3.2) pg/mL, while the median (min-max) value in the K2 group was 198.61 (74.12-1548.82) pg/mL. The Mann-Whitney test showed a significant difference in PDGF levels between the two groups before treatment with a $p$-value $=0.014(p<0.05)$, indicating a different baseline condition of the PDGF levels of both groups and it was able to affect the results. This study randomly selected subjects who met the inclusion and exclusion criteria in two groups by simple random sampling, so that several conditions that could cause false increases in PDGF levels were avoided. Platelet-derived growth factor levels can increase in conditions of wound healing, pulmonary

Table 1. Data on characteristics of research subjects

\begin{tabular}{lccc}
\hline \multirow{2}{*}{ Characteristics } & \multicolumn{2}{c}{ Group } & \multirow{2}{*}{ p-value } \\
\cline { 2 - 3 } & Control (K1) & Treatment (K2) & \\
\hline Gender & & & $0.303^{\pi}$ \\
Male & $6(35.3 \%)$ & $10(58.8 \%)$ & \\
Female & $11(64.7 \%)$ & $7(41.2 \%)$ & \\
Age (years) & $42.29 \pm 12.11$ & $40.88 \pm 13.02$ & $0.746^{\star}$ \\
Weight (kg) & $56.29 \pm 8.74$ & $53.00 \pm 6.19$ & $0.125^{\star}$ \\
Height (cm) & $164.29 \pm 3.5$ & $165.06 \pm 3.77$ & $0.545^{\star}$ \\
Albumin (g/dL) & $2.89 \pm 0.53$ & $2.80 \pm 0.51$ & $0.622^{\star}$ \\
BMI & $17.95(35.3 \%)$ & $17.29(41.2 \%)$ & $0.543^{€}$ \\
Low & $21.66(52.9 \%)$ & $20.95(58.8 \%)$ & \\
Normal & $25.65(11.8 \%)$ & $0(0 \%)$ & \\
High & $12(70.6 \%)$ & $12(70.6 \%)$ & $0.897^{*}$ \\
Surgery & $3(17.6 \%)$ & $4(23.5 \%)$ & \\
Laparotomy & $1(5.9 \%)$ & $1(5.9 \%)$ & \\
Cholecystectomy & $1(5.9 \%)$ & $0(0 \%)$ & \\
Cystectomy & & \\
Myomectomy & & \\
\hline
\end{tabular}

*Independent sample T-test (unpaired, normal data distribution) \ Chi-Square test (unpaired, nominal data) $€$ Kruskal-Wallis test (unpaired, abnormal distribution)

$¥$ Mann-Whitney test (unpaired, ordinal data)

Table 2. Comparison of PDGF levels in K1 and K2 group before treatment

\begin{tabular}{|c|c|c|c|}
\hline \multirow{2}{*}{ Variable } & K1 & K2 & \multirow{2}{*}{$\mathbf{p}$} \\
\hline & Mean (SD) & Median (min-max) & \\
\hline PDGF (pg/mL) & $561.55(3.2)$ & $198.61(74.12-1548.82)$ & $0.014 *$ \\
\hline $\begin{array}{l}\text { PDGF = Platelet-Derived Grc } \\
\text { median = data was not norm }\end{array}$ & uted & $\begin{array}{l}\text { was normally distributed } \\
\text { ney test }\end{array}$ & $\mathrm{SD}=$ Standard Deviation \\
\hline
\end{tabular}


Results of PDGF levels measurement after treatment in the $\mathrm{K} 1$ group showed a median (min-max) value of 758.93 (38.02-3162.28) $\mathrm{pg} / \mathrm{mL}$, whereas in the $\mathrm{K} 2$ group the mean (SD) was 467.94 (2.9) $\mathrm{pg} / \mathrm{mL}$. the Mann-Whitney test showed no significant difference in PDGF levels between the two groups after treatment with a p-value $=0.344$ $(p>0.05)$. There was an increase in PDGF levels in the two groups on the fourth day because the wound healing process can cause an increase in PDGF levels. Wound healing begins when PDGF is released from the platelets and stored in the wound during the blood clotting process, then causes chemotaxis of leukocytes, monocytes and fibroblasts which play a role in the duration of the inflammatory response, stimulation of fibroblasts proliferation, connective tissue formation, epithelial regeneration, capillary formation and development of scars. ${ }^{7}$ Comparison of PDGF levels between $\mathrm{K} 1$ and $\mathrm{K} 2$ group after treatment is shown in Table 3.

Wilcoxon test was used to determine differences in PDGF levels before and after treatment in the K1 group. There was no significant difference in PDGF levels in the $\mathrm{K} 1$ group before and after treatment with a $p$-value $=0.653(p>0.05)$. This result was in accordance with the research by Szabo et al. in 2007 in Hungary that there was no significant difference in serum PDGF levels in patients during the initial postoperative period tested before surgery and the fourth post-operative day $(p=0.248) .{ }^{14}$ A comparison of serum PDGF levels before and after treatment in the $\mathrm{K} 1$ group is shown in Table 4.

The Wilcoxon test was used to determine differences in PDGF levels before and after treatment in the K2 group (for pairs). The results showed that there were significant differences in the PDGF levels in the K2 group before and after treatment with a value of $p=<0.001(p<0.05)$. One of the contents of

Table 3. Comparison of PDGF levels between $\mathrm{K} 1$ and $\mathrm{K} 2$ group after treatment

\begin{tabular}{llll}
\hline \multirow{2}{*}{ Variable } & \multicolumn{1}{c}{ K1 } & K2 & p \\
\cline { 2 - 3 } & Median (min-max) & Mean (SD) & $0.344^{*}$ \\
\hline PDGF pg/mL) & $758.93(38.02-3162.28)$ & $467.94(2.9)$ & \multirow{2}{*}{} \\
\hline
\end{tabular}

PDGF = Platelet-Derived Growth Factor median = data was not normally distributed mean = data was normally distributed $S D=$ Standard Deviation *Mann-Whitney test

Table 4. Comparison of PDGF levels in $\mathrm{K} 1$ group before and after treatment

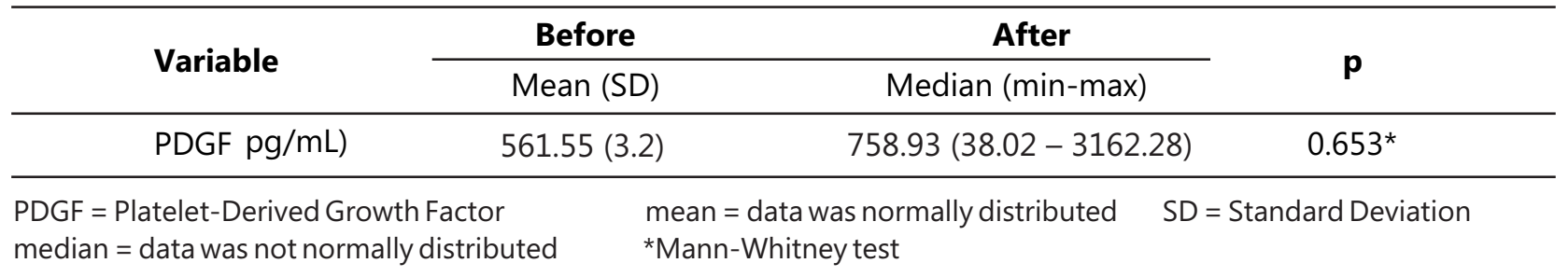

Table 5. Comparison of PDGF levels in the $\mathrm{K} 2$ group before and after treatment

\begin{tabular}{llll}
\hline \multirow{2}{*}{ Variable } & Before & After & p \\
\cline { 2 - 3 } & Median (min-max) & Mean (SD) & $<0.001^{*}$ \\
\hline PDGF pg/mL) & $198.61(74.12-1548.82)$ & $467.94(2.9)$ & $<$ \\
\hline
\end{tabular}

PDGF = Platelet-Derived Growth Factor median = data was not normally distributed mean = data was normally distributed $S D=$ Standard Deviation *Mann-Whitney test

Table 6. Comparison of delta PDGF levels in K1 and K2 group

\begin{tabular}{lccc}
\hline \multirow{2}{*}{ Variable } & K1 & K2 & \multirow{2}{*}{} \\
\cline { 2 - 3 } & Median $($ min-max $)$ & Median $($ min-max $)$ & \\
\hline PDGF pg/mL) & $232.27(-3421.1-1997.1)$ & $214.96(-17-2663)$ & $0.438^{\star}$ \\
\hline
\end{tabular}

PDGF = Platelet-Derived Growth Factor median = data was not normally distributed mean = data was normally distributed $\quad S D=$ Standard Deviation

*Mann-Whitney test 
snakehead fish and sea cucumber extract was an unsaturated fatty acid which regulates prostaglandin synthesis. Prostaglandin acts as a blood vessel vasodilator that regulates neutrophil infiltration and activation (the role of PDGF released by $\alpha$-platelet granules) in the inflammatory process and induces healing wound, significantly increased the PDGF levels of the subject after treatment on the fourth day.

A comparison of serum PDGF levels before and after treatment in the $\mathrm{K} 2$ group is shown in Table 5.

A Mann-Whitney test was used to determine differences in delta PDGF levels between the two study groups. There was no significant difference in delta PDGF levels between K1 and K2 groups, with a value of $p=0.438(p>0.05)$ as shown in Table 6 .

It was concluded that standard therapy and the administration of snakehead fish and sea cucumber extract were able to significantly increase PDGF levels on the fourth post-operative day which showed a better effect on wound healing because it speeded up the healing process.

\section{CONCLUSION AND SUGGESTION}

Based on the results of this study it was found that supplementation with snakehead fish and sea cucumber extract was able to increase PDGF levels in postoperative patients.

Snakehead fish and sea cucumber fish extracts should be given before surgery to get optimal results. Measurement of PDGF levels can be used to monitor post-operative wound healing because PDGF plays a role in each phase of wound healing.

\section{REFERENCES}

1. Park SA, Raghunathan VK, Shah NM, Teixeira L, Motta MJ, et al. PDGF-BB does not accelerate healing in diabetic mice with splinted skin wounds. PLoS One, 2014; 9(8): e104447.

2. Mohd J, Syah Y, Omar E, Pai DR, Sood S. Cellular events and biomarkers of wound healing. Indian J Plast Surg, 2012; 45(2): 220-8.

3. Demoulin JB, Almendras C. Platelet-derived growth factors and their receptors in normal and malignant hematopoiesis, Am J Blood Res, 2012; 2(1): 44-56.

4. Sun BK, Siprashvili Z, Khavari PA. Advances in skin grafting and treatment of cutaneous wounds. Science, 2014; 346, 941.

5. Schmidt MB, Chen EH, Lynch SE. A review of the effects of insulin-like growth factor and platelet derived growth factor on in-vivo cartilage healing and repair, Osteo Arthritis and Cartilage 14, 2006; $403 \mathrm{e} 412$.

6. Tahara A, Yasuda M, Itagane $H$, Toda I, Teragaki $M$, et al. Plasma levels of platelet-derived growth factor in normal subjects and patients with ischemic heart disease. American Heart Journal, 1991; 122(4 pt 1): 986-92.

7. Ross R. Platelet-derivet growth factor. Ann Rev Med, 1987; 38: 71-79.

8. Liu G, Gurung AS, Qiu W. Lateral flow aptasensor for simultaneous detection of Platelet-Derived Growth Factor-BB (PDGF-BB) and thrombin. Molecules, 2019; 24(756): 1-11.

9. Wahab SZA, Kadir AA, Hussain NHN, Omar J, Yunus $R$, et al. The effect of Channa striatus (Haruan) extract on pain and wound healing of post-lower segment caesarean section women. Hindawi Publishing Corporation, 2015; 849647: 6.

10. Bordbar S, Anwar F, Saari N. High-value components and bioactives from sea cucumbers for functional foods. Marine Drugs, 2011; 9: 1761-1805.

11. Hanna VS, Hafez EAA. Synopsis of arachidonic acid metabolism. Journal of Advanced Research, 2018; 11:23-32.

12. Safri M, Manan A. Therapeutic potential of the Haruan (Channa striatus): From food to medicinal uses. Malaysian Journal of Nutrition, 2012; 1: 18.

13. Mohamed IN, Mazlan M, Shuid AN. Sea cucumber (stichopus chloronotus) to expedite healing of minor wound. International Conference on Biomedical Engineering, 2015; 9: 11.

14. Szabó A, Laki J, Madsen HO, Dósa E, Proh `aszka Z, et al. Early rise in serum VEGF and PDGF levels predisposes patients with a normal MBL2 genotype to restenosis after eversion endarterectomy. Stroke AHA Journals, 2007; 38: 2247-2253. 